\title{
Evaluation of the buccal component of systemic absorption with inhaled fluticasone propionate
}

\author{
Owen J Dempsey, Wendy J R Coutie, Andrew M Wilson, Peter Williams, Brian J Lipworth
}

\begin{abstract}
Background-Inhaled corticosteroids have dose related systemic effects determined by oral (swallowed or oropharyngeal absorption) and lung bioavailability. A study was undertaken to evaluate the significance of oropharyngeal absorption for fluticasone propionate.

Methods-Sixteen healthy volunteers of mean age 29.3 years were studied using an open randomised, placebo controlled, four way crossover design. Treatments were: (a) fluticasone metered dose inhaler (pMDI) $250 \mu \mathrm{g}, 8 \mathrm{puffs}$; (b) fluticasone pMDI $250 \mu \mathrm{g}, 8$ puffs + mouth rinsing/ gargling (water); (c) fluticasone pMDI $250 \mu \mathrm{g}, 8$ puffs + mouth rinsing/gargling (charcoal); and (d) placebo pMDI, 8 puffs + mouth rinsing/gargling (water). Overnight (ONUC) and early morning (EMUC) urinary cortisol/creatinine ratios and 8 am serum cortisol (SC) levels were measured.
\end{abstract}

Results-Significant $(\mathrm{p}<0.05)$ suppression of ONUC, EMUC, and SC occurred with each active treatment compared with placebo. The mean values (95\% CI for difference from placebo) were: (a) ONUC (nmol/mmol): fluticasone $(2.8,95 \%$ CI 3.6 to 7.9$)$, fluticasone + water $(3.1,95 \%$ CI 3.3 to 7.7$)$, fluticasone + charcoal $(2.3,95 \%$ CI 4.1 to 8.5); placebo (8.6); (b) EMUC (nmol/mmol): fluticasone (5.6, 95\% CI 8.4 to 24.5$)$, fluticasone + water $(7.6,95 \%$ CI 6.6 to 22.4); fluticasone + charcoal (5.6, 95\% CI 8.7 to 24.5); placebo (22.1). There were no significant differences between active treatments. The numbers of subjects with an overnight urinary cortisol of <20 nmol/10 hours were 0 (placebo), 11 (fluticasone), 12 (fluticasone + water), and 13 (fluticasone + charcoal).

Department of Clinical

Pharmacology \&

Therapeutics,

Ninewells Hospital \&

Medical School,

University of Dundee,

Dundee DD1 9SY, UK

O J Dempsey

W J R Coutie

A $M$ Wilson

$\mathrm{P}$ Williams

B J Lipworth

Correspondence to:

Professor B J Lipworth.

Received 28 January 1999

Returned to author

24 March 1999

Revised manuscript received

7 April 1999

Accepted for publication

7 April 1999

Keywords: adrenal suppression; inhaled corticosteroids; asthma; fluticasone propionate

Inhaled corticosteroids are currently recognised as first line anti-inflammatory therapy in patients with asthma. ${ }^{1}$ It is recognised, how- ever, that all inhaled corticosteroids are associated with dose related systemic adverse effects, being absorbed into the systemic circulation via the gastrointestinal tract (oral bioavailability) or the lung (lung bioavailability). ${ }^{2}$

Oral bioavailability is determined firstly by the fraction of drug systemically absorbed across the oropharyngeal mucosa and, secondly, by the swallowed fraction which undergoes first pass hepatic metabolism. Absorption from the lung, as from the buccal cavity, is not subject to first pass metabolism. The degree of hepatic first pass metabolism differs among the inhaled corticosteroids. ${ }^{3}$

Fluticasone propionate exhibits almost complete $(99 \%)$ first pass inactivation in the liver. ${ }^{4}$ The systemic bioavailability of fluticasone propionate is therefore determined mainly by its lung bioavailability as well as any potential oropharyngeal absorption. It is well recognised that, when using a pressurised metered dose inhaler (pMDI), the majority of inhaled corticosteroid (approximately 80\%) is deposited in the oropharynx with only $10-20 \%$ reaching the lung. It is therefore important to know whether there is a significant degree of direct buccal absorption and whether this moiety can be attenuated by mouth rinsing.

The main purpose of this study was therefore to dissect out the lung versus oropharyngeal components of systemic absorption when using fluticasone via pMDI alone. As there is almost complete first pass metabolism of the swallowed fraction, any reduction in systemic absorption due to mouth rinsing can be assumed to be due to the buccal component per se. We therefore employed a rigorous mouth rinsing protocol using both activated charcoal and water.

\section{Methods}

SUBJECTS

Sixteen healthy non-smoking volunteers (eight men) were recruited into the study. Their mean (SE) age was 29.3 (2.3) years. The subjects were all non-smokers with no history of respiratory or other disease. In addition, all had normal physical examination, spirometric indices, urinalysis and routine haematology/ biochemical blood tests. Pregnant women and those on the oral contraceptive pill were excluded. Approval for the study was obtained from the Tayside medical ethics committee and all patients gave written informed consent.
This was an open label single centre, placebo controlled, randomised study with a four way crossover design. Subjects attended an initial screening during which they were instructed in 
Table 1 Mean values with 95\% confidence intervals for each treatment compared with placebo

\begin{tabular}{|c|c|c|c|c|}
\hline & Placebo & Fluticasone & Fluticasone + water & Fluticasone + charcoal \\
\hline 8.00 am serum cortisol $(\mathrm{nmol} / \mathrm{l})$ & 425.3 & $202.0(130.7$ to 316.0$)$ & $241.5(91.2$ to 276.5$)$ & $186.0(146.7$ to 332.0$)$ \\
\hline Overnight urinary cortisol/creatinine $(\mathrm{nmol} / \mathrm{mmol})$ & 8.6 & $2.8(3.6$ to 7.9$)$ & $3.1(3.3$ to 7.7$)$ & $2.3(4.1$ to 8.5$)$ \\
\hline Early morning urinary cortisol/creatinine $(\mathrm{nmol} / \mathrm{mmol})$ & 22.1 & 8.4 to 24.5$)$ & 7.6 ( 6.6 to 22.4 ) & 8.7 to 24.5$)$ \\
\hline
\end{tabular}

the correct use of a pMDI using a Vitalograph aerosol inhalation monitor device (Vitalograph, Bucks, UK). Subjects were not permitted to proceed into the study unless they had good technique by "passing" the training test. Assessment of inhaler technique was also reinforced at each subsequent laboratory visit.

There were four separate laboratory visits, each separated by at least a five day washout period. All treatments were administered under supervision at the same time of day. All subjects took all of the following treatment protocols in a randomly selected order: (a) placebo pMDI: eight consecutive puffs in conjunction with mouth rinsing and gargling with water (as a total of $400 \mathrm{ml}$ divided into eight aliquots of $50 \mathrm{ml}$ after each puff); (b) fluticasone pMDI $250 \mu \mathrm{g}$ : eight consecutive puffs without mouth rinsing or gargling; (c) fluticasone pMDI $250 \mu \mathrm{g}$ : eight consecutive puffs in conjunction with mouth rinsing and gargling with water (as a total of $400 \mathrm{ml}$ divided into eight aliquots of $50 \mathrm{ml}$ after each puff); (d) fluticasone pMDI $250 \mu \mathrm{g}$ : eight consecutive puffs in conjunction with mouth rinsing and gargling with activated charcoal (as $50 \mathrm{~g}$ of Carbomix (Penn Pharmaceuticals Ltd, Gwent UK) suspended in a total volume of $400 \mathrm{ml}$ water divided into eight aliquots of $50 \mathrm{ml}$ after each puff).

Gargling and rinsing took place for 30 seconds after actuation of the inhaler and the fluid was discarded (rather than swallowed).

\section{MEASUREMENTS}

After completion of the inhalation sequence the subjects went home with written instructions to collect an overnight urinary cortisol sample from $10 \mathrm{pm}$. Subjects were asked to empty their bladder at $10 \mathrm{pm}$, collecting all voided urine until they returned to the laboratory the next day at $7.30 \mathrm{am}$. They were asked to lie supine for 30 minutes before taking an 8.00 am plasma cortisol sample. Following this, subjects voided another sample of urine at $8.00 \mathrm{am}$ to complete the 10 hour collection. The volume of the total sample was recorded and aliquots were taken for overnight (10 hour) and early morning $(8.00 \mathrm{am})$ urinary cortisol and creatinine measurements.

ASSAYS

All assays were performed in duplicate in a blinded fashion by a separate technician. Serum cortisol was measured with a commercial RIA kit (Incstar Ltd, Wokingham, Berkshire, UK) which has no cross reactivity with fluticasone propionate. The coefficient of variation (CV) for analytical imprecision for serum cortisol was $5.5 \%$ within the assay and $10.9 \%$ between the assay. For urinary free cortisol excretion the within assay $\mathrm{CV}$ was $7.9 \%$ and the between assay CV was $12.1 \%$. Urinary creatinine was measured on a Cobas-bio auto- analyser (Roche Products Ltd, Welwyn Garden City, Herts, UK). The intra-assay $\mathrm{CV}$ was $1.92 \%$ and the inter-assay CV was $4.9 \%$.

Data analysis

The study was powered at $80 \%$ with a sample size of 16 in order to detect a $20 \%$ difference in overnight urinary cortisol excretion. All data were analysed with a Statgraphics software package (STSC Software Group, Rockville, Maryland, USA). All data were normally distributed and analysed parametrically. Comparisons were made by a multifactorial analysis of variance (MANOVA) using subject, treatment, and period as factors. This was followed by Bonferroni multiple range testing set at $95 \%$ confidence interval in order to obviate multiple pairwise comparisons. Hence, all comparisons are reported as being significant $(\mathrm{p}<0.05$, two tailed) or not. $95 \%$ confidence intervals for differences between treatments were calculated.

\section{Results}

Significant $(\mathrm{p}<0.05)$ suppression of overnight or early morning urinary cortisol/creatinine ratio and serum cortisol levels occurred with each treatment containing fluticasone propionate compared with placebo (table 1, fig 1).
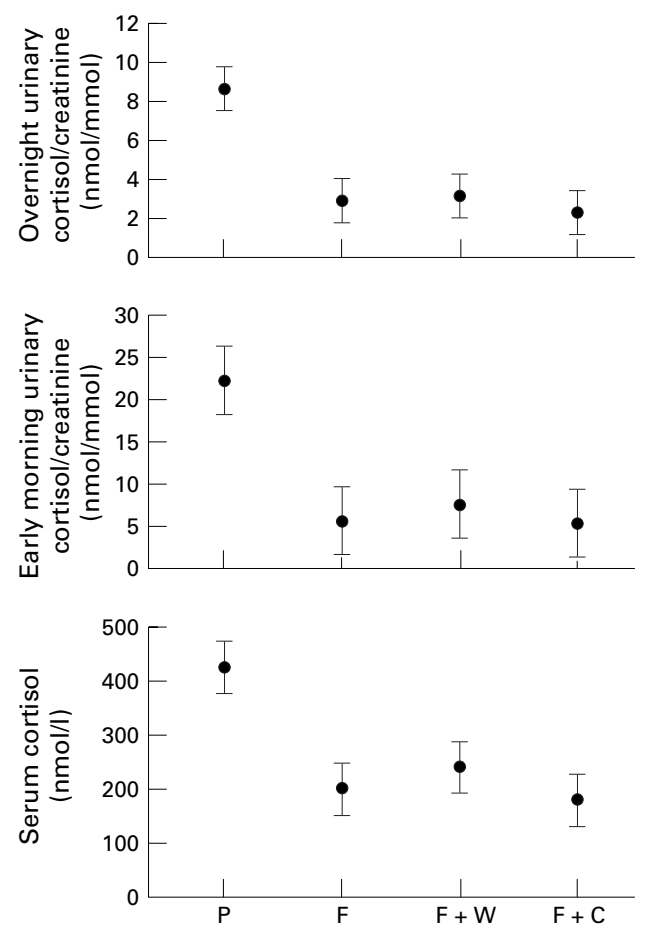

Figure 1 Mean overnight and early morning urine cortisol/creatinine ratios, and early morning serum cortisol levels for each treatment. Error bars represent $95 \%$ confidence intervals from pooled SE values. All three active treatments were significantly $(p<0.05)$ different from placebo for all three end points. $P=$ placebo, $F=$ fluticasone propionate, $W=$ water mouth rinse + gargle, $C=$ charcoal mouth rinse + gargle. 


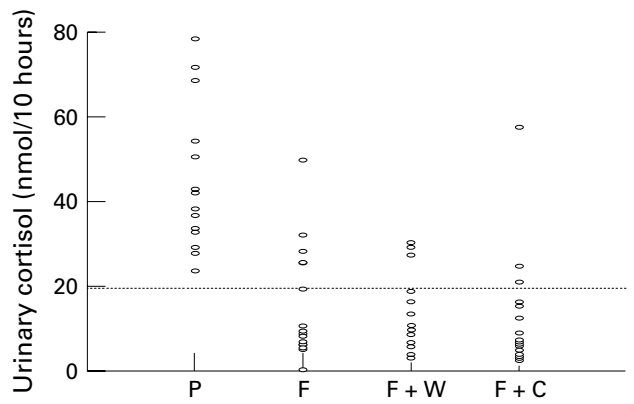

Figure 2 Individual values for uncorrected overnight urinary cortisol excretion for each treatment. Dotted line represents cut off value of 20 nmol/10 hours. $P=$ placebo, $F$ = fluticasone propionate, $W=$ water mouth rinse + gargle, $C=$ charcoal mouth rinse + gargle .

No significant differences were observed in the mean degree of adrenal suppression between fluticasone propionate alone or in combination with vigorous mouth washing with either water or activated charcoal. Individual data for uncorrected urinary free cortisol levels are shown in fig 2 to illustrate dispersion and outliers. No subjects in the placebo group had urinary cortisol excretion suppressed to below $20 \mathrm{nmol} / 10$ hours compared with 11 subjects given fluticasone only, 12 subjects given fluticasone + water, and 13 subjects given fluticasone + charcoal mouth wash.

\section{Discussion}

Our study is the first to assess the contribution of oropharyngeal absorption of inhaled fluticasone propionate towards adrenal suppression, a surrogate marker of overall systemic bioavailability. ${ }^{5}$ It is now clear using charcoal block that absorption of fluticasone across the oropharyngeal mucosa does not contribute significantly to the overall systemic bioactivity profile. Furthermore, gargling with water in conjunction with use of fluticasone via a pressurised metered dose inhaler does not lead to reduced systemic bioactivity.

Fluticasone propionate is a suitable corticosteroid for assessing the buccal absorption moiety for several reasons. Firstly, because of $99 \%$ first pass metabolism in the liver, ${ }^{46}$ even if our patients had inadvertently swallowed any corticosteroid this would be unlikely to reach the systemic circulation and be implicated in subsequent adrenal suppression. Any adrenal suppression seen can therefore be assumed to be secondary to absorption of fluticasone directly from the lung, assuming the effectiveness of activated charcoal in attenuating buccal absorption. ${ }^{7}$ Secondly, fluticasone propionate has a high degree of lipophilicity relative to other inhaled corticosteroids, ${ }^{8}$ thus ensuring good mucosal absorption. Thirdly, we have previously found a marked degree of detectable systemic bioactivity via a pMDI, as assessed by sensitive markers of adrenal suppression, with similar doses of fluticasone propionate. ${ }^{910}$

Measuring adrenal suppression is a recognised way of assessing systemic bioactivity of an inhaled corticosteroid. ${ }^{5}$ An overnight or first waking collection of urinary free cortisol is simple for the patient to collect and is as sensi- tive as a 24 hour collection, particularly when corrected for creatinine excretion. ${ }^{112}$

Using activated charcoal as a mouth wash effectively removes possible oropharyngeal absorption from the oral bioavailability equation and allows assessment of the relative gut and lung components of systemic bioavailability. ${ }^{3}$ No differences were observed in the degree of adrenal suppression associated with the use of rigorous mouth washing (water or charcoal) between each inhalation of fluticasone. Our results suggest that direct buccal absorption of fluticasone propionate is not an important component of systemic bioactivity, which is more likely to be due to absorption across the lung vascular bed of the inhaled fraction. Intuitively, this is perhaps not surprising, given the relatively small absorptive surface area of the buccal mucosa compared with the lung and the short mucosal exposure time before swallowing occurs.

Mouth washing with water is unlikely therefore to reduce the systemic bioactivity of fluticasone propionate. This may not apply to other corticosteroids such as beclomethasone dipropionate or budesonide due to their lower first pass hepatic inactivation, estimated to be $90 \%$ and $70 \%$, respectively. ${ }^{13}{ }^{14}$ Studies examining the effect of mouth rinsing on systemic bioactivity of these corticosteroids have produced conflicting results. In one study mouth rinsing was associated with a $15 \%$ difference in serum cortisol suppression with $1.6 \mathrm{mg}$ budesonide daily via a Turbohaler ${ }^{15}$ whilst another study suggested mouth rinsing had no impact on 24 hour urinary free cortisol in patients taking $1.6 \mathrm{mg}$ budesonide daily via Turbohaler for two weeks. ${ }^{16}$ Following a single $2 \mathrm{mg}$ dose of beclomethasone by Diskhaler, mouth rinsing without swallowing also did not appear to influence adrenal suppression (as 8.00 am serum cortisol), perhaps reflecting the use of only $20 \mathrm{ml}$ of water after the last inhalation. ${ }^{17}$ Despite this, the same authors noted in another study that swallowing $50 \mathrm{~g}$ of activated charcoal after a single dose of $2 \mathrm{mg}$ belomethasone by Diskhaler reduced 8.00 am serum cortisol suppression by $48 \% .^{7}$ This result is in keeping with the known low degree of hepatic first pass metabolism of beclomethasone. Nevertheless, whatever the inhaled corticosteroid, mouth washing with water after use may be of value to obviate local complications such as buccal candidiasis, particularly when using higher doses. This study was supported by a University of Dundee
departmental grant. The authors wish to thank Mrs L $\mathrm{McF}$ arlane for performing the biochemical assays.

1 British Thoracic Society, et al. The British guidelines on asthma management: 1995 review and position statement. Thorax 1997;52(Suppl 1):S1-21.

2 Lipworth BJ. New perspectives on inhaled drug delivery and systemic bioactivity. Thorax 1995;50:105-10.

3 Lipworth BJ. Pharmacokinetics of inhaled drugs. $\mathrm{Br} 7 \mathrm{Clin}$ Pharmacol 1996;42:697-705.

4 Harding SM. The human pharmacology of fluticasone propionate. Respir Med 1990;84(Suppl A):25-9.

5 Lipworth BJ, Seckl JR. Measures for detecting systemic bioactivity with inhaled and intranasal corticosteroids. Thorax 1997;52:476-82.

6 Thorsson L, Dahlstrom K, Edsbacker A, et al. Pharmacokinetics and systemic effects of inhaled fluticasone propionate in healthy subjects. Br f Clin Pharmacol 1997;43:15561 . 
7 Trescoli-Serrano, Ward MJ, Rajput R, et al. Does swallowed charcoal affect gastrointestinal absorption of inhaled becolcharcoal affect gastrointestinal absorption of inh
methasone? Eur Respir f 1995;8 (Suppl 19):304s.

8 Johnson M. Development of fluticasone propionate and comparison with other inhaled corticosteroids. F Allergy Clin Immunol 1998;101:S434-9.

9 Clark DJ, Grove A, Cargill RI, et al. Comparative adrenal suppression with inhaled budesonide and fluticasone propionate in adult asthmatic patients. Thorax. 1996;51 262-6.

10 Wilson AM, McFarlane LC, Lipworth BJ. Dose-response effect for adrenal suppression with repeated twice daily inhaled fluticasone propionate and triamcinolone acetonide in adult asthmatics. Am $\mathcal{F}$ Respir Crit Care Med 1997; 156:1274-7.

11 Wilson AM, Lipworth BJ. 24 hour and fractionated profiles of adrenocortical activity in asthmatic patients receiving in-

2 McIntyre DH, Mitchell CA, Bowler SD, et al. Measuring the systemic effects of inhaled beclomethasone: timed morning urine collections compared with 24 hour specimens. Thorax 1995;50:1280-4.

13 Martine LE, Harrison C, Tanner RJN. Metabolism of beclomethasone dipropionate by animals and man. Postgrad Med F 1975;51(Suppl 4):11-20.

14 Ryrfeldt A, Andersson P, Edsbacker S, et al. Pharmacokinetics and metabolism of budesonide, a selective glucocorticoid. Eur F Respir Dis 1982;63(Suppl):s86-95.

15 Selroos O, Halme $M$. Effect of a volumatic spacer and mouth rinsing on systemic absorption of inhaled corticosteroid from a metered dose inhaler and dry powder inhaler. Thorax 1991;46:891-4.

16 O'Connor B, Aikman S, Edsbacker S, et al. The influence of mouth washing on the systemic effects of budesonide turbuhaler. Am f Respir Crit Care Med 1997;155(Suppl): A355.

17 Trescoli-Serrano C, Ward MJ, Chu S, et al. Does mouth rinsing influence the systemic availability of inhaled beclomethasone? Eur Respir f 1995;8(Suppl 19):304. 